\title{
Towards reconciling structure and function in the nuclear pore complex
}

\author{
Roderick Y. H. Lim · Ueli Aebi · Birthe Fahrenkrog
}

Accepted: 7 December 2007 / Published online: 29 January 2008

(C) Springer-Verlag 2008

\begin{abstract}
The spatial separation between the cytoplasm and the cell nucleus necessitates the continuous exchange of macromolecular cargo across the double-membraned nuclear envelope. Being the only passageway in and out of the nucleus, the nuclear pore complex (NPC) has the principal function of regulating the high throughput of nucleocytoplasmic transport in a highly selective manner so as to maintain cellular order and function. Here, we present a retrospective review of the evidence that has led to the current understanding of both NPC structure and function. Looking towards the future, we contemplate on how various outstanding effects and nanoscopic characteristics ought to be addressed, with the goal of reconciling structure and function into a single unified picture of the NPC.
\end{abstract}

\section{Introduction}

The cytoplasmic and nuclear compartments of eukaryotic cells are separated by the nuclear envelope (NE). The double membrane of the NE is perforated by nuclear pore complexes (NPCs), which are large multiprotein complexes that support passive diffusion of small molecules, and facilitate receptor-mediated translocation of proteins and ribonucleoprotein complexes. Overall, the vertebrate NPC is a $\sim 120$ MDa protein complex made of $\sim 30$ different proteins called nucleoporins (or Nups) that are repetitively arranged as distinct subcomplexes to form the NPC (Cronshaw et al. 2002; Lim and Fahrenkrog 2006; Rout et al. 2000;

R. Y. H. Lim $(\bowtie) \cdot$ U. Aebi · B. Fahrenkrog M.E. Müller Institute for Structural Biology, Biozentrum, University of Basel, Klingelbergstrasse 70, Basel 4056, Switzerland e-mail: roderick.lim@unibas.ch
Schwartz 2005; Tran and Wente 2006). In the plane of the NE, the eightfold symmetric central framework of the NPC, also known as the spoke complex, encloses a central pore that is $\sim 50 \mathrm{~nm}$ long and is narrowest $(\sim 40 \mathrm{~nm})$ at the NE midplane (Beck et al. 2004, 2007; Stoffler et al. 2003). Attached to the central framework are cytoplasmic filaments and a nuclear basket (Figs. 1,2).

We begin, here, with a retrospective that summarizes the analysis and elucidation of NPC architecture by electron microscopy (EM) techniques. We will discuss how the dissection of NPC structure by electron tomography and X-ray crystallography has in a methodical and progressive manner reached the ultrastructural, molecular and atomic scale. In parallel, we will overview the progress that has contributed to the contemporary understanding of how the NPC functions as a selective gate-barrier. Setting our sights on the future, we will close by highlighting the importance of reconciling both NPC structure and function, as we strive towards converging on a single conceptual, mechanistic understanding of the NPC.

\section{Nuclear pore complex architecture}

NPCs are the most distinctive structural components of the NE as resolved by EM. NPC structure was initially dissected using transmission EM (TEM), but later expanded to scanning transmission as well as to scanning EM and, most recently, to cryo-electron tomography (CET). The first EM study on the NE in 1950 revealed that it is perforated by pores (Callan and Tomlin 1950). Gall (1967) showed for the first time that NPCs exhibit an octagonal structure (Fig. 1a). This was later confirmed by a number of studies in the following years (Aaronson and Blobel 1974; Franke and Scheer 1970; Maul 1971), whereby its overall architecture, 
particularly its eightfold symmetry, appeared to be evolutionarily conserved (Franke and Scheer 1970). Although its functional significance was ambiguous at that time, it was already evident from these early studies that the NPC is composed of a "membranous" structure, specifically with a part residing within the NE, and nonmembranous, filamentous structures being attached to the cytoplasmic and the nuclear face of the NPC (Fig. 1b) (Franke and Scheer 1970; Kessel 1969).

According to today's consensus, the NPC consists of an approximately cylindrical central framework, eight cytoplasmic filaments and a nuclear basket (Fig. 1c-g) that is composed of eight filaments that join into a distal ring (Fig. 2b, d, f). Refinement in EM techniques using negatively stained and frozen-hydrated NPCs from Xenopus laevis oocyte NEs (Akey and Radermacher 1993; Hinshaw et al. 1992; Unwin and Milligan 1982) (Fig. 2a) or frozenhydrated yeast cells (Fig. 2c) (Yang et al. 1998) provided early $3 \mathrm{D}$ reconstructions of the central framework. The central framework of the NPC (also known as the spoke complex) resides within the NE, and is anchored to the region where the inner and outer nuclear membranes fuse. Early structural studies showed that the cytoplasmic and nuclear ring moieties are integral to the central framework. Recent CET studies on isolated nuclei from Xenopus oocytes (Stoffler et al. 2003) and intact, transport-competent nuclei isolated from Dictyostelium discoideum (Fig. 2f) (Beck et al. 2004, 2007) have improved the resolution of the central framework to $6 \mathrm{~nm}$ and revealed the first reconstructions of peripheral, flexible components of the NPC, i.e. the cytoplasmic filaments and the nuclear basket. In Dictyostelium, the cytoplasmic filaments are about $\sim 35 \mathrm{~nm}$ in length and the nuclear basket is about $60 \mathrm{~nm}$ long. Together with the $\sim 50 \mathrm{~nm}$ central framework, the NPC has an overall length of about $150 \mathrm{~nm}$ and an outer diameter of $125 \mathrm{~nm}$ (Beck et al. 2004, 2007). Although the overall linear dimensions of the NPC vary between species, the overall 3D architecture appears to be evolutionarily conserved (Fahrenkrog et al. 1998; Kiseleva et al. 2004; Yang et al. 1998).

Enclosed by the central framework is the hourglassshaped central pore of the NPC, which has a diameter of
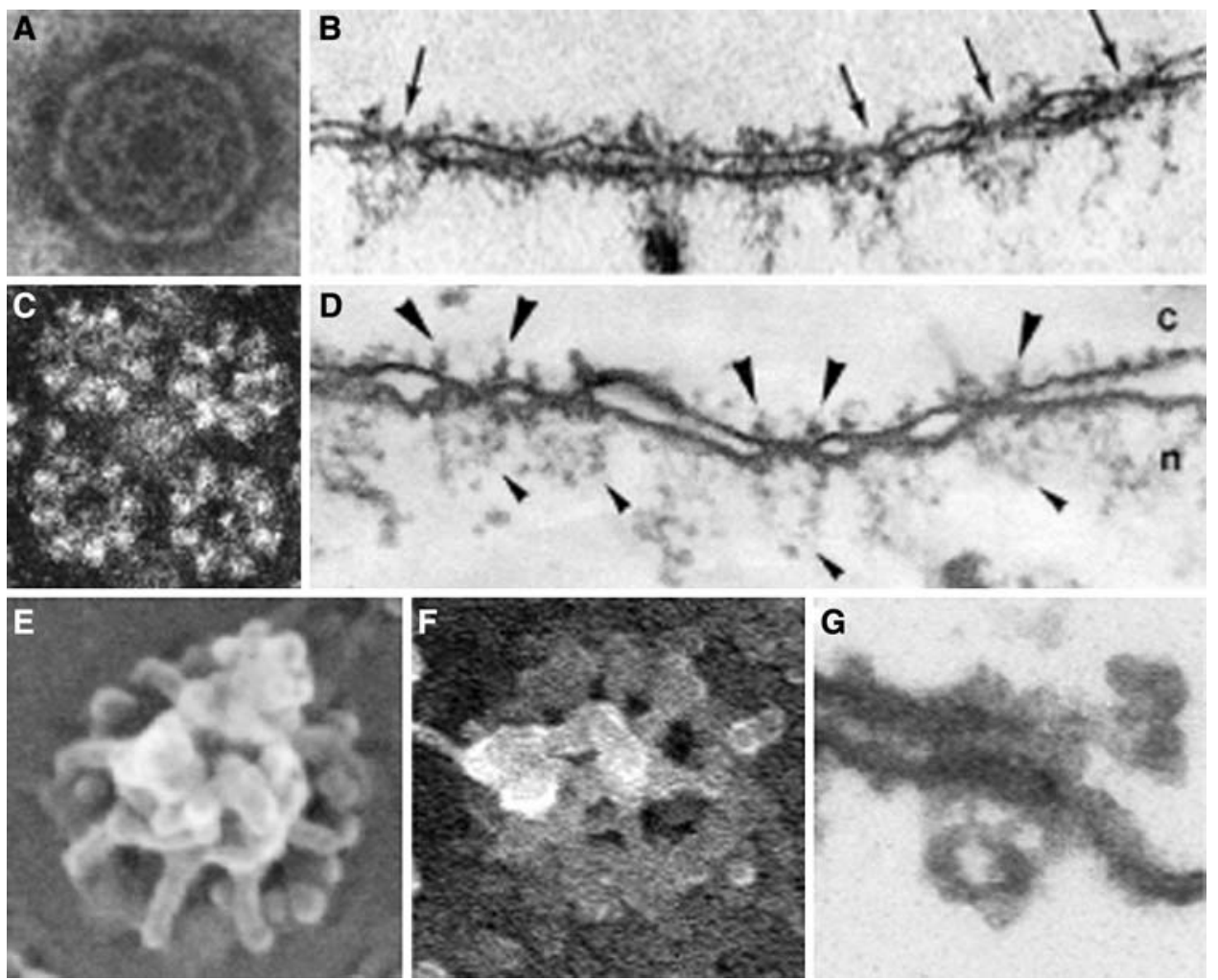

Fig. 1 Electron micrographs of NPCs over time. a Cytoplasmic face of negatively stained and $\mathbf{b}$ a stretch along a nuclear envelope of embedded and thin-sectioned nuclei from Triturus alpestrus. c Cytoplasmic face of negatively stained and $\mathbf{d}$ a stretch along a nuclear envelope of embedded and thin-sectioned nuclei from Xenopus laevis. View of the nuclear basket of isolated nuclei from Xenopus oocytes prepared by e critical point drying and field emission scanning electron microscopy, f quick-freeze/freeze-drying/rotary metal shadowing and $\mathbf{g}$ thin-sectioning and transmission electron microscopy. a Reproduced with permission from Gall (1967). b Reprinted with permission from Franke and Scheer (1970). c Reprinted with permission from Gerace and Burke (1988). d Reprinted with permission from Pante and Aebi (1996). e Courtesy of Martin Goldberg (www.dur.ac.uk/m.w.goldberg/). f Courtesy of Bohumil Maco (University of Queensland, Australia). g Courtesy of Birthe Fahrenkrog 

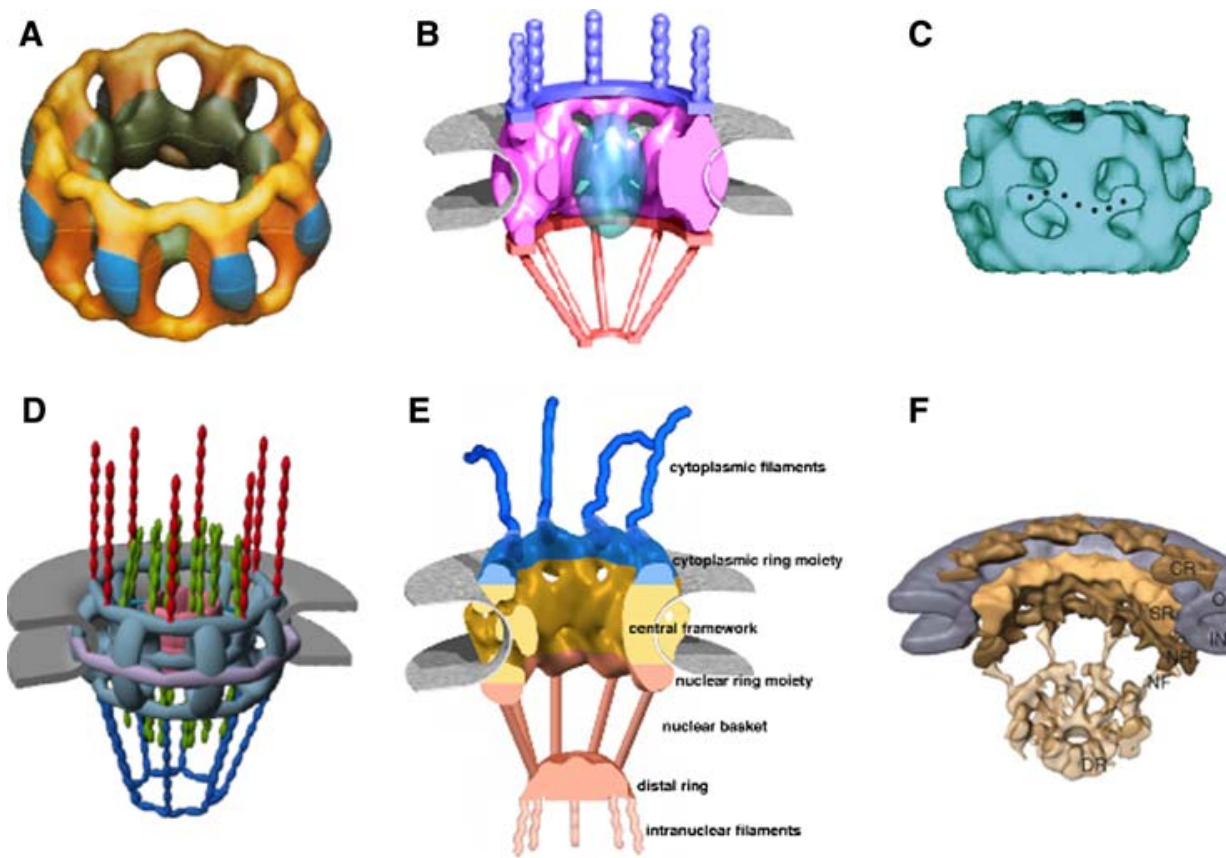

$\mathbf{F}$

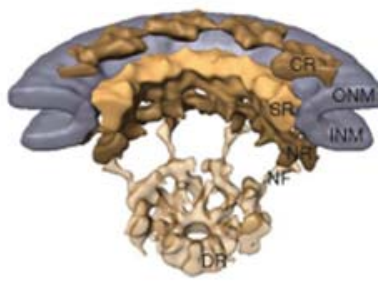

Fig. 2 NPC models in chronological order. Three-dimensional reconstruction of the central framework of negatively stained NPCs after detergent treatment $\mathbf{a}$ from Xenopus oocytes, and $\mathbf{b}$ its adaptation for a consensus model of the NPC architecture and $\mathbf{c}$ from yeast nuclei and $\mathbf{d}$ its adaptation into a model. e Consensus model of the NPC based on a reconstruction of native NPCs embedded in thick amorphous ice. f Reconstruction of NPCs from intact nuclei of Dictyostelium discoid- eum examined by cryo-electron tomography. a Reprinted with permission from Hinshaw et al. (1992). b Reprinted with permission from Pante and Aebi (1995). c Reprinted with permission from Yang et al. (1998). d Reprinted with permission from Rout and Aitchison (2001). e Reproduced from Fahrenkrog and Aebi (2003). f Reproduced with permission from Beck et al. (2007)

1992; Stoffler et al. 2003). Other potential roles for the peripheral channels have been proposed, such as in maintaining the NE electrical conductance (Danker et al. 1999; Enss et al. 2003; Mazzanti et al. 2001; Shahin et al. 2001) or as mechanical buffer zones that accommodate deformations of the central framework upon translocation of large cargoes (Stoffler et al. 2003).

The number of NPCs per cell varies greatly with cell size and activity. Yeast cells have $\sim 200$ NPCs/nucleus, proliferating human cells have $\sim 10-20 \mathrm{NPCs} / \mu \mathrm{m}^{2}$ (i.e. 2,000-5,000 NPCs/nucleus) and a mature Xenopus oocyte has about $60 \mathrm{NPCs} / \mu \mathrm{m}^{2}$ yielding $\sim 5 \times 10^{7} \mathrm{NPCs} /$ nucleus (Gerace and Burke 1988; Gorlich and Kutay 1999). A comprehensive ultrastructural study using freeze-fracture EM of yeast cells in combination with $3 \mathrm{D}$ reconstruction has shown that the distribution of yeast NPCs in the NE is not equidistant, but rather cluster into regions of higher density (Winey et al. 1997). This observation is not limited to yeast and is present in other cell types as well (Franke 1974). In yeast, the number of NPCs was found to increase steadily, beginning in the G1-phase and peaking in the S-phase of the cell cycle, suggesting that NPC assembly occurs continuously throughout the cell cycle (Winey et al. 1997). Similarly, the density of NPCs increases throughout the cell cycle in HeLa S3 cells (Maeshima et al. 2006). 


\section{Biochemical composition of the nuclear pore complex: the nucleoporins}

The molecular building blocks of the NPC comprise 30 different proteins known as nucleoporins or Nups, which are present in at least eight copies per NPC (Cronshaw et al. 2002; Rout et al. 2000). Based on secondary structure prediction, nucleoporins can be grouped into three distinct classes (Devos et al. 2006). The transmembrane group, which contains transmembrane $\alpha$-helices and a cadherin fold, comprises the outermost features of the NPC central framework and is thought to assist in anchoring the NPC to the NE. The second group of nucleoporins contain $\beta$-propeller and $\alpha$-solenoid folds, which localizes towards the inside of the NPC, whereas the third class harbors the conserved sequence motif of phenylalanine-glycine (FG)repeats in combination with a coiled-coil fold and likely contributes to the formation of the NPC's inner central framework and the peripheral structures (Devos et al. 2006; Schwartz 2005; Tran and Wente 2006). Other less frequent structural motifs found in nucleoporins are zinc-finger domains as in Nup153 and RanBP2/Nup358 (Higa et al. 2007) or RNA-recognition motifs as in Nup35 (Handa et al. 2006).

$\beta$-Propellers are predicted in the third class of the nucleoporins, and in fact seven-bladed $\beta$-propellers have been resolved from the N-terminal domains (NTD) of the human nucleoporins Nup133 and Nup214 as well as from the NTD of Nup159p in yeast by X-ray crystallography (Berke et al. 2004; Napetschnig et al. 2007; Weirich et al. 2004). Proteins with $\beta$-propeller folds participate in diverse cellular functions and serve as platforms for multiple dynamic protein-protein interactions. Along this line, yeast Nup133p and Nup159p both play roles in mRNA export from the nucleus, given that deletion or mutations in their NTDs impair their functions in mRNA export, probably by preventing the association of multiple mRNA export factors with the NPC (Berke et al. 2004; Weirich et al. 2004).

The NTD of human Nup133 furthermore contains an amphipathic $\alpha$-helical motif capable of sensing membrane curvature (Drin et al. 2007). This motif corresponds to an exposed loop, which connects two blades of the $\beta$-propeller and folds to an $\alpha$-helix upon interacting with small liposomes. Whether the $\alpha$-helical motif in Nup133 serves to recognize the curved topology of the nuclear pore membrane to anchor the NPC during interphase or to recognize small vesicles containing $\mathrm{NE}$ fragments critical for $\mathrm{NE}$ reassembly after mitosis, or both, remains to be clarified (Drin et al. 2007). The NTD of human Nup214, in comparison to its yeast homolog Nup159p, consists of two distinct structural elements: the $\beta$-propeller and a 30-residue C-terminal extended peptide segment (Napetschnig et al. 2007).
This extension binds to the bottom of the $\beta$-propeller with low affinity and has been suggested to play an "auto-inhibitory" role in NPC assembly.

The first crystal structure obtained for a nucleoporin was the NPC targeting domain of human Nup98 (Hodel et al. 2002). This domain, similar to the nuclear pore-targeting domain of its yeast homolog Nup116p, consists of a six-stranded $\beta$-sheet sandwiched against a two-stranded $\beta$-sheet and is flanked by two $\alpha$-helical regions (Hodel et al. 2002; Robinson et al. 2005). This domain exhibits multiple conformations and is stabilized only when bound to a ligand, i.e. Nup96 and Nup145p-C in the case of Nup98 and Nup116p, respectively (Robinson et al. 2005). Conformational diversity may allow Nup98 and Nup116p to bind to multiple targets within the NPC or to associate and dissociate quickly from the NPC to increase the mobility of the nucleoporins, as described for Nup98, which shuttles in a transcription-dependent manner (Griffis et al. 2002, 2004).

An attempt to crystallize the first subcomplex of the NPC, the Nup62 complex, yielded the structure of the $\alpha$-helical coiled-coil domain of rat Nup58/45 (Melcak et al. 2007). Nup58/45 forms tetramers in the crystal structure consisting of two antiparallel dimers. Each dimer consists of two $\alpha$-helices that are connected by a short loop. The intradimer interface is hydrophobic, whereas dimer-dimer interactions occur through large hydrophilic residues. The tetramer can adopt various conformations leading to a lateral displacement between tetramers suggesting an intermolecular sliding mechanism (Melcak et al. 2007). The Nup62 complex has recently been mapped to the cytoplasmic periphery of the NPC's central pore (Schwarz-Herion et al. 2007), so that sliding of Nup58/45, and possibly of Nup62 and Nup54 as well, could contribute in modulating the diameter of the central pore in response to transport activity (Melcak et al. 2007).

FG-repeat domains (also known as FG-domains) are found in about one-third of the nucleoporins and mediate the interaction between soluble transport receptors loaded with signal-bearing cargo and the NPC. These FG-domains constitute the key components of the selective gate-barrier that limits the diffusion of cargoes through the NPC (see following section). Atomic structures of short FG-repeat peptides in complex with, for example, the import receptor importin $\beta$ (Bayliss et al. 2000, 2002b), NTF2 (Bayliss et al. 2002a) or the putative mRNA export factor TAP/ NXF1 (Grant et al. 2002, 2003), have consistently shown that the interaction between FG-repeats and the different transport receptors involves primarily the phenylalanine ring of the FG-repeat core and hydrophobic residues on the surface of the receptor (Isgro and Schulten 2005, 2007a, b). Hydrophilic linker regions between individual FG-motifs, which constitute the majority of amino acid mass in the 
overall FG-domain, appear to influence the strength of the binding and allow simultaneous binding of several FGcores to the receptor (Liu and Stewart 2005).

Based on biophysical measurements, the FG-domains of yeast nucleoporins have been found to be natively unfolded (Denning et al. 2002, 2003), i.e. having little or no secondary structure. Similarly, FG-domains of human, fly, worm and other yeast species are most likely disordered based on their amino acid composition (Denning and Rexach 2007). This notion is further supported by immuno-EM studies of vertebrate FG-repeat nucleoporins, which suggest that the FG-domains are flexible and mobile within the NPC (Fahrenkrog et al. 2002; Paulillo et al. 2005, 2006; Schwarz-Herion et al. 2007). Single molecule studies using atomic force microscopy (AFM) on the recombinantly expressed FGdomain of human Nup153 further revealed that this $\sim 700$ residue domain is in fact an $\sim 180 \mathrm{~nm}$ long unfolded molecule when adsorbed on the surface of mica (Lim et al. 2006a). Based on the eightfold rotational symmetry of the NPC, it is now estimated that each NPC is populated by at least 128 FG-domains together displaying 3,500 FG-repeats (Strawn et al. 2004).

Nup153 and Nup214 are both known to play roles in distinct nucleocytoplasmic transport (NCT) pathways and interact with a number of nuclear transport receptors via their FG-repeats (Ball and Ullman 2005; Bernad et al. 2006; Hutten and Kehlenbach 2006; Sabri et al. 2007; van Deursen et al. 1996). The location of the FG-domains of Nup153 and Nup214 shifts in a transport-dependent manner in the NPC, further supporting their role in NCT (Paulillo et al. 2005). Systematic deletion of FG-repeat regions in yeast nucleoporins revealed, however, that yeast NPCs suffer from only slight changes in distinct nuclear transport pathways, but otherwise remain functional despite having removed up to $50 \%$ of their FG-repeats (Strawn et al. 2004). This suggests that the FG-repeats exhibit a functional redundancy in bulk NCT, except for specific nuclear transport pathways, which may require individual FGnucleoporins, and/or that other interaction sites for transport receptors exist within the NPC (Terry and Wente 2007).

Besides playing important roles in NCT, certain FGrepeats have been implicated in other functions as well. The crystal structure of the RRM domain of mouse Nup35 revealed that all three FG-sequences of this nucleoporin are in ordered secondary structure elements and that these FGsequences do not interact with transport receptors, such as importin $\beta$, but rather with, for example, the integral membrane protein Ndc1. Thus, the FG-sequences of Nup35 may contribute to the formation of the NPC's central framework (Handa et al. 2006).

\section{Nucleocytoplasmic transport and nuclear pore complex function}

NPCs are porous to small molecules (e.g. water and ions), which freely diffuse through the NPC, while more massive cargoes (i.e. $>40 \mathrm{kDa}$ in size) [a recent study has showed that this limit may extend to $\sim 100 \mathrm{kDa}$ (Wang and Brattain 2007)] require the assistance of soluble transport receptor proteins to be effectively chaperoned through the NPC. A large number of these receptor proteins, known collectively as karyopherins (Kaps) or more specifically as importins (imp) and exportins (exp), were discovered around the 1990s (Rexach and Blobel 1995; Wozniak et al. 1998) [most recently reviewed in (Stewart 2007)]. Today, the biochemical role of the receptors in "unlocking" the NPC barrier as part of the nuclear trafficking machinery is relatively well understood. As illustrated in Fig. 3, appropriate cargoes are identified through a short sequence of residues known as nuclear localization/export signals (i.e. NLS/NES) for import and export, respectively, which exhibit binding interactions with the karyopherins [sometimes using an adaptor such as, for example, importin- $\alpha$ (Gorlich et al. 1994)]. Otherwise, passage through the NPC is obstructed for large, non-NLS/NES

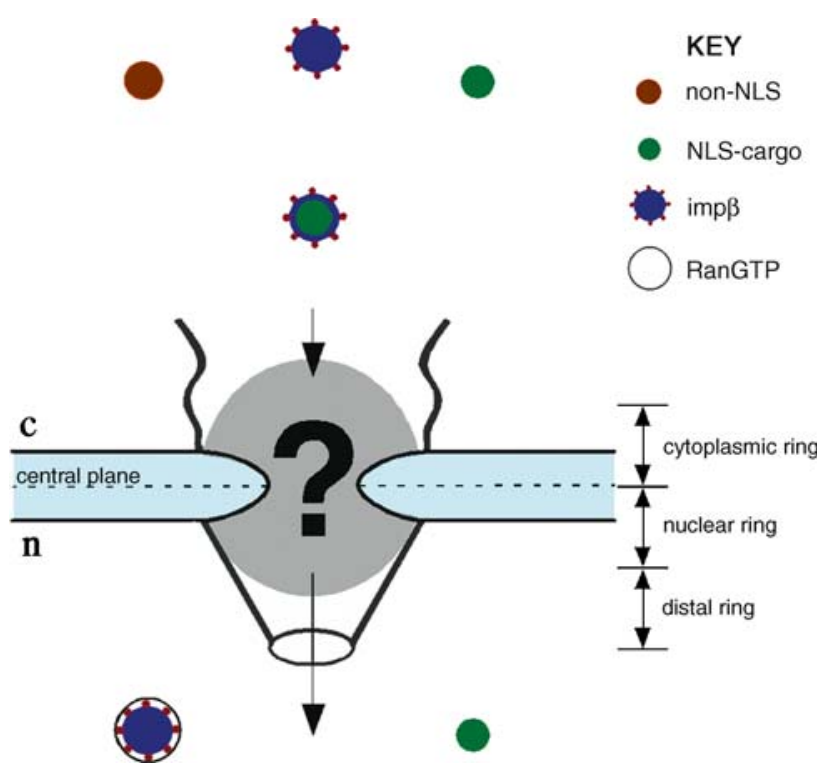

Fig. 3 Schematic representation identifying the main biochemical constituents of the nuclear import machinery. Passage through the NPC is restricted to transport receptors, which bind and chaperone NLS-cargo through the NPC, while access is prohibited for passive (non-NLS) molecules of similar size. Binding of RanGTP to the transport receptor releases the NLS-cargo into the nucleus. The gray shaded area emphasizes the location of the FG-domains and the question mark highlights the uncertainty with regard to the biophysical aspects of the selective gating mechanism within the NPC, which operates to restrict or promote cargo translocation 
harboring molecules that do not bind to the karyopherins (i.e. passive) (Paine et al. 1975). The directionality of NCT is driven by an asymmetric distribution of the two nucleotide states of the small GTPase Ran (Melchior et al. 1993; Moore and Blobel 1993). Being predominant in the nucleus, RanGTP functions to release the cargo from its import receptor by binding to the receptor itself (Gorlich et al. 1996). Within the nucleus, exportins bind to their cargo in the presence of RanGTP, which ferries the complex back into the cytoplasm. Once in the cytoplasm, GTP hydrolysis causes the disassembly of the export complex, thereby recycling the export receptor and fueling the cytoplasmic RanGDP pool.
A

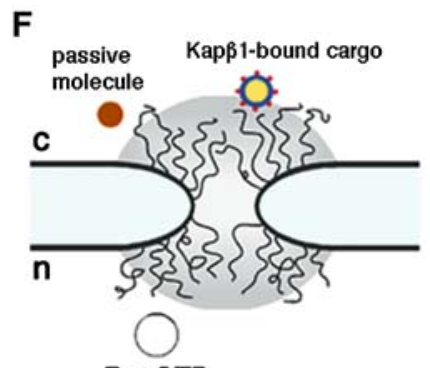

RanGTP

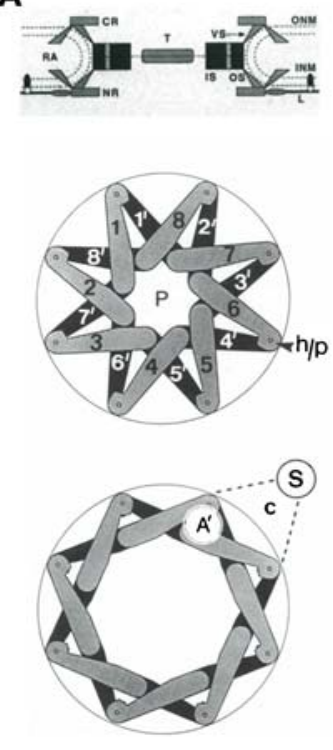

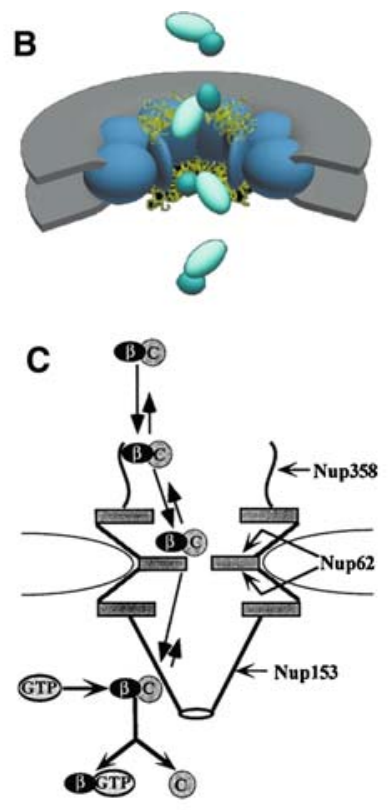

D

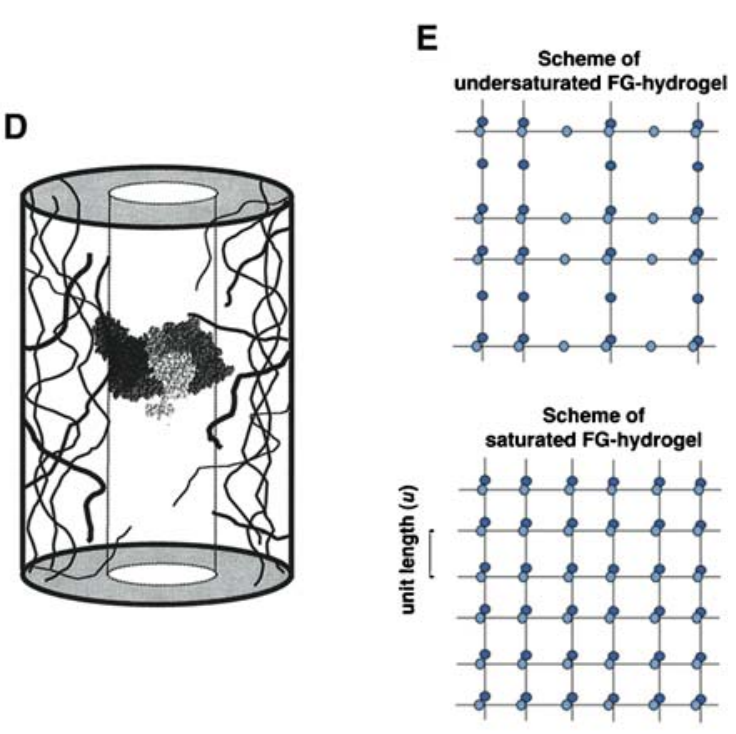

E

Scheme of
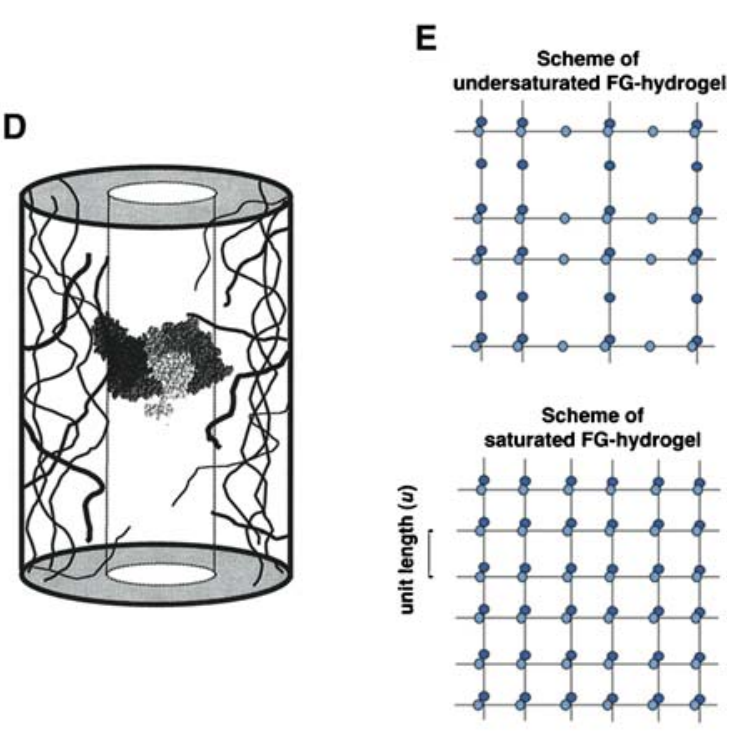

Early models of the NPC gating mechanism were derived from initial EM studies, which linked the biophysical origin " the selective gate to the presence of a "central plug" or 1989, 1990). The central tansporter was suggested to consist of an iris-like mechanism that is hinged to the central channel (Akey 1990) (Fig. 4a). Although recent evidence indicates that this feature represents to a large extent cargo complexes arrested during translocation (Beck et al. 2004, 2007; Stoffler et al. 2003), this model suggested a translocation mechanism that has largely defined the

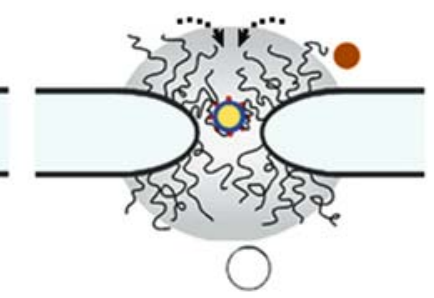

Fig. 4 Getting to the bottom of the NPC selective gating mechanism. a The central transporter consisting of an iris-like mechanism that is hinged to the central channel. b Being natively unfolded and located at the nuclear/cytoplasmic peripheries of the NPC, the virtual gating model suggests that the entropic movements of the FG-domains can act as a barrier to inert cargo. $\mathbf{c}$ The affinity gradient model proposes that receptor-cargo complexes traverse a pathway that is lined by FGdomains of increasing affinity. $\mathbf{d}$ The oily-spaghetti model predicts that the FG-domains can only be pushed aside by receptor-cargo complexes. e The undersaturated and subsequently the saturated hydrogel model purport that the FG-domains crosslink via a dense

\section{Modeling NPC function} . 
criteria for subsequent NPC models in the field (Akey and Goldfarb 1989). These steps include a peripheral binding to the NPC, followed by a docking step, and then a translocation step (e.g. by a dilation of the transporter).

Coupled to the requirement of Kaps, Radu et al. (1995b) recognized that the FG-domains represent the key constituents of the NPC selective gate by determining that the FGrepeats act as docking sites for Kaps. By identifying and binding to the FG-domains, the Kaps provide the biochemical selection mechanism for NCT (Radu et al. 1995a, b). Consequently, Rout et al. (2000) showed that each NPC consists of up to 12 different FG-domains (i.e. nucleoporins), which are located near the nuclear and cytoplasmic peripheries of the NPC. To further emphasize the functional redundancy between the various FG-domains in the NPC (1) the asymmetric FG-Nups have been shown to be dispensable for NCT (Zeitler and Weis 2004); (2) the direction of transport through the NPC can be inverted (Nachury and Weis 1999); (3) active transport is able to proceed in NPCs lacking cytoplasmic filaments (i.e. FG-rich Nup358) (Walther et al. 2002); (4) the selective gating mechanism has been found to remain functional even after $50 \%$ of the FGrepeats had been depleted (Strawn et al. 2004).

Taken together, it is generally agreed that the FGdomains act as the physical constituents of the NPC barrier (Ben-Efraim and Gerace 2001; Macara 2001; Ribbeck and Gorlich 2002; Rout et al. 2000) (Fig. 3). However, the mechanistic manner in which the FG-domains contribute to the selective gating of the NPC is widely speculated and has been the subject of several reviews (Fahrenkrog and Aebi 2003; Lim et al. 2006a; Stewart 2007; Suntharalingam and Wente 2003; Weis 2003). Akin to the entropic fluctuations of unstructured microtubule-associated proteins (MAPs) (Mukhopadhyay and Hoh 2001) and neurofilament sidearms (Brown and Hoh 1997), the Brownian affinity gating model (Rout et al. 2000), later called virtual gating (Rout et al. 2003), proposes that the entropic behavior of peripheral FG-domains acts as a substantial barrier to inert cargo (Fig. 4b). Translocation is anticipated for receptormediated cargoes due to interactions between the FGrepeats and the transport receptors, which increases the residence time and probability of entry into the NPC. In a similar manner, the "oily-spaghetti" model (Macara 2001) postulates that noninteracting FG-domains are pushed aside by cargo complexes but otherwise obstruct the passage of passive cargo (Fig. 4d).

Beyond its barrier function, the dualistic ability of each NPC to restrict or promote cargo translocation (i.e. to simultaneously act as a barrier and a vectorial transport facilitator) is only figuratively understood. Karyopherincargo complex movement through the NPC has been compared to "stepping from one FG-repeat to the next" (Rexach and Blobel 1995), or "sliding over a surface comprised of
FG-repeats" (Peters 2005). Alternatively, the affinity gradient model (Ben-Efraim and Gerace 2001) suggests that transport complexes "step" through NPCs lined with FGnucleoporins exhibiting increasing binding affinities with the cargo complexes (Fig. 4c). Finally, the selective phase model (Ribbeck and Gorlich 2002) predicts that FGdomains attract each other via hydrophobic inter-FG-repeat interactions to form a hydrophobic gel or meshwork (Fig. 4e). This interpretation is based on experiments, which show that the addition of hydrophobic solvents disrupts the meshwork and triggers a nonselective opening of the pore (Ribbeck and Gorlich 2002; Shulga and Goldfarb 2003). Hence, it is predicted that passive, more hydrophilic material is obstructed while hydrophobic cargo complexes are able to "dissolve" through the sieve-like meshwork.

\section{Unraveling FG-domain behavior}

Frey et al. (2006) showed that the yeast FG-Nup, Nsp1, can be cast in the form of a macroscopic hydrogel to lend support to the "selective phase" model (Ribbeck and Gorlich 2002). However, the same authors reported that the Nsp1 hydrogel lacked any discriminatory effects between inert, non-Kap binding proteins and Kap-complexed proteins unless the FG concentration within the gel was raised ( $\sim 50 \mathrm{mM})$ to the point where it formed a "saturated" hydrogel (Frey and Gorlich 2007). In this manner, they explained that an efficient permeability barrier was recovered due to the formation of a highly ordered meshwork consisting of cohesive inter-FG repeats (Fig. 4e). In addition to preventing the translocation of inert molecules, it was found that such a saturated hydrogel could reproduce the diffusion rates of receptor-driven transport in the NPC (Kubitscheck et al. 2005; Yang et al. 2004).

To address this issue, Patel et al. (2007) reported evidence that refutes the gel-forming quality of Nsp1. To investigate the cohesiveness of different FG-domains, Patel et al. devised a low affinity assay, which could detect the binding of CFP-Nups to GST-Nups immobilized on Sepharose beads. By avoiding the non-physiological conditions used to form the hydrogels (Frey et al. 2006; Frey and Gorlich 2007), they found that only GLFG-domains show weak cohesive interactions, whereas FxFG-domains (such as Nsp1) do not bind together. Based on their findings, the authors suggested that the FxFG domains on both cytoplasmic and nuclear peripheries act as an entropic repulsive barrier, while the GLFG-domains form a cohesive meshwork in the central NPC channel (Patel et al. 2007). Therefore, they proposed a two-gate model that combines elements of both Brownian gating and the selective phase (Patel et al. 2007). Such a two-gate model, however, is likely to be more appropriate to yeast NPCs, because verte- 
brate NPCs are known to be composed almost entirely of FxFG-domains (except for the GLFG-domain of Nup98) (Suntharalingam and Wente 2003). Furthermore, by a systematic depletion of FG-domains in yeast, the authors showed that the NPCs displayed similar qualitative "leakiness" in all the cases studied, which indicates that all the FG-domains exhibit a functional redundancy in that both peripherally and centrally anchored FG-Nups play equal roles in maintaining the selective gating mechanism.

Lim et al. (2006b, 2007a) developed an experimental platform that allowed for the collective behavior of surfacetethered FG-domains to be probed at the nanoscopic length scale. By reproducing the physical dimensions of the NPC, they found that the FxFG-domains of the vertebrate Nup153 resembles a polymer brush (Halperin et al. 1992; Milner 1991; Zhao and Brittain 2000). This is caused by packing constraints between the entropy-dominated FGdomains, which force the surface-tethered FG-domains to extend in a net directionality away from the surface (i.e. entropic barrier). This creates a significant repulsive barrier that can repel large macromolecules while allowing small molecules such as ions and water to diffuse more easily through. This may explain why a reduced number of FGdomains is enough to maintain an effective barrier in the NPC. By showing that the extended brush-like FG-domains collapsed in hexanediol, they were further able to explain why the NPCs could reversibly open and close when similar reagents were added/removed in nuclear transport assays (Patel et al. 2007; Ribbeck and Gorlich 2002; Shulga and Goldfarb 2003). This was further substantiated with atomic force microscope (AFM) single molecule force spectroscopy (SMFS) analysis, which showed that individual Nup153 FG-domain molecules (1) lack intra-FG interactions; (2) are natively unfolded; (3) can be reversibly stretched and relaxed without any change to its intrinsic entropic elasticity, i.e. resembling a worm-like chain (WLC) (Bustamante et al. 1994; Marko and Siggia 1995). In comparison, SMFS analysis revealed complex plateaulike (un)binding topologies between $\operatorname{Kap} \beta 1$ (importin $\beta$ ) and Nup153 when Kap $\beta 1$-modified AFM tips were used (Lim et al. 2007b). This provided evidence for binding promiscuity (Dunker et al. 2001; Tompa 2002) in FG-receptor interactions and is in agreement with the fact that $\operatorname{Kap} \beta 1$ consists of five experimentally verified hydrophobic FGbinding sites (Bayliss et al. 2000, 2002b; Bednenko et al. 2003; Liu and Stewart 2005), with additional five binding sites predicted by molecular dynamics (MD) simulations that can be simultaneously occupied (Isgro and Schulten 2005).

To further correlate the governing biochemical interactions to the biophysical behavior of the FG-domains, Lim et al. (2007a) studied the nanomechanical response of the brush-like FG-domains under the influence of transport fac- tors such as Kap $\beta 1$, RanGTP and RanGDP. Consequently, they found that the entropic barrier collapsed in vitro due to Kap $\beta 1$-FG binding interactions (i.e. causing a reduction in conformational entropy of the FG-domains) and was reversed by the sequestration of $\operatorname{Kap} \beta 1$ by RanGTP (but not RanGDP). By validating similar effects in situ using an immunogold-EM labeling assay in Xenopus oocytes nuclei, they hypothesized that the selective gating mechanism consists of a rapid, stochastic flux of collapsing and distending FG-domains that regulates passage through the NPC (Fig. 4f). Such "seamlessness" may explain the reversibility of NCT and apparent open communication between the cytoplasm and nucleus (Kopito and Elbaum 2007).

\section{Outlook}

Besides resolving the overall structure of the NPC, an accurate picture of how selective gating is achieved by the (structureless) FG-domains remains unclear due to a general lack of understanding with regard to their behavior and function within the NPC. So far, only the reversible collapse of the FG-domains has been directly observed to occur in the NPC (Lim et al. 2007a). The source of this ambiguity stems in part from the difficulty in trying to visualize the FG-domains in vivo, which is evident given the lack of resolution even when using state-of-the-art structural techniques such as cryo-tomography (cryo-ET) to detect the FG-domains (Beck et al. 2004, 2007; Stoffler et al. 2003). Although immunogold-EM techniques can provide positional information of the FG-domains within the NPC, this is limited to static views of FG-domain behavior (Fahrenkrog et al. 2002; Paulillo et al. 2005, 2006; Schwarz-Herion et al. 2007; Lim et al. 2007a). New microscopic techniques such as the AFM is also usually limited in resolution and chemical sensitivity due to the complexity of the NPC and its cellular environment (Jaggi et al. 2003a, b; Mooren et al. 2004; Stoffler et al. 1999; Wang and Clapham 1999). In fact, owing to their natively unfolded structure (Denning et al. 2002, 2003), the FG-domains themselves have so far only been directly visualized as individual bioploymers by AFM (Lim et al. 2006a).

In combination with biochemical efforts to identify whether different transport receptors use preferential FGdomains during NCT (Terry and Wente 2007), it may be beneficial to use bottom-up strategies to investigate how the FG-domains behave on a biophysical level to give rise to the selective gating of the NPC. This is because biochemical approaches can only provide a marginal mechanistic description of the selective gating process. Moreover, because NCT occurs over tens of nanometers in and around the NPC, i.e. a near-field effect, this in fact necessitates a consideration of such physical details, including (1) the 
geometry and nanoscopic dimensions of the NPC, (2) the FG-domain anchoring sites within the NPC and (3) the limited number of FG-domains located within the NPC.

Thus, it will be essential to establish FG-domain conformations at the NPC-relevant length scale. To critically underscore this point, can macroscopic assays that address the characteristics of enormously large numbers of FGdomains be interpreted to represent and describe the biophysical behavior of $\sim 100-150$ natively unfolded FGdomains anchored within the NPC? How does one reconcile the notion of having extended FG-domains in the NPC when the diameter of the central pore is about ten times larger than the size of a FG-domain at dynamic equilibrium [Stokes' radius typically a few nanometers (Denning et al. 2003)]? What physical basis can allow for the FG-domains on opposing sides of the central pore to extend so far past their Stokes' radius so as to "touch each other," much less to form an inter-FG crosslinked meshwork spanning the diameter of the central pore (Frey and Gorlich 2007) (Fig. 4e), in a highly vacuous NPC (Beck et al. 2004, 2007)? In fact, can the presence of strong FG-FG binding effects prevent the formation of such a meshwork by causing individual FG-domains to collapse or "curl up" along the walls of the central channel (Weis 2007)? Conversely, if a brush-like conformation is a valid prerequisite for the extension of the FG-domains to form a barrier in the NPC (Lim et al. 2006b), does this necessarily preclude the possibility of inter-FG interactions occurring between neighboring FG-domains?

Bearing in mind that the FG-domains are anchored to the NPC instead of free floating in solution, whether or not FXFG- and GLFG-domains cohere in the NPC (Patel et al. 2007) will not only depend on how strongly (and dynamically, i.e. frequently) they interact with each other, but also on their physical proximity within the NPC (i.e. anchoring site). Therefore, other contextual effects that require consideration include the following: (1) How confinement effects based on the hourglass-like geometry affect the entropic behavior of the FG-domains (e.g. preferring to remain in the central pore or outside)? (2) The strength of FG-FG interactions in the midst of competing effects such as the steric hindrance between the hydrophilic regions of neighboring FG-domains; (3) How nonspecific interactions can bias their behavior (Paradise et al. 2007; Timney et al. 2006), e.g. macromolecular crowding (Zimmerman and Minton 1993)? (4) More generally, how does the complex cellular environment (exhibiting a multitude of specific/ nonspecific interactions) in and around the NPC near-field affect FG-domain conformations? (5) Finally, how do these effects influence and affect the interactions between the FGdomains and transport receptors?

Given these arguments, the modus operandi of the NPC selective gating mechanism requires careful substantiation at the near-field, mesoscopic scale (i.e. the length scale relevant to the properties of a material or phenomenon). To emphasize its relevance, the outermost surface of a hydrogel at the gel-liquid interface has been shown to consist of noncrosslinked polymer chains (Kim et al. 2001, 2002). Moreover, FG-domain behavior will have to be assessed at the relevant time scales, since selective gating appears to be a rapid kinetic process over a time scale of the order of $\sim 5 \mathrm{~ms}$ (Kubitscheck et al. 2005; Yang et al. 2004; Yang and Musser 2006). This emphasizes the need to move beyond conventional biological methods and to adopt more interdisciplinary experimental approaches (Dutta and Belfort 2007). In addition, the use of molecular dynamics (MD) simulations (Isgro and Schulten 2005, 2007a, b) and other theoretical frameworks (Zilman et al. 2007) may be able to illuminate aspects of FG-domain behavior and the nuclear trafficking machinery that experimentalists can then look out for and validate.

Perhaps most importantly, the desire to conceptually reconcile and reach a single objective understanding of NPC function and structure will require that such aspects of nuclear transport processes be scrutinized in individual NPCs in vivo. However, beyond the technical challenges involved, a difficulty remains as to how a "ground-state" can be defined in the NPC-so as to monitor subsequent changes in the nebulous haze of FG-domains-without any quantitative information regarding the endogenous receptors and cargo already bound to the FG-domains, which will influence FG-domain behavior. In any case, such attention to detail will be key to defining a unified picture of the NPC. As noted by Paine et al. (1975) in Nature more than 30 years ago, "as solute size approaches the dimensions of the pore, solute-pore wall interactions become increasingly important. Specific site interactions... would also influence solute movements."

\section{References}

Aaronson RP, Blobel G (1974) On the attachment of the nuclear pore complex. J Cell Biol 62:746-754

Akey CW (1989) Interactions and structure of the nuclear-pore complex revealed by cryo-electron microscopy. J Cell Biol 109:955-970

Akey CW (1990) Visualization of transport-related configurations of the nuclear-pore transporter. Biophys J 58:341-355

Akey CW, Goldfarb DS (1989) Protein import through the nuclearpore complex is a multistep process. J Cell Biol 109:971-982

Akey CW, Radermacher M (1993) Architecture of the xenopus nuclear-pore complex revealed by 3-dimensional cryoelectron microscopy. J Cell Biol 122:1-19

Ball JR, Ullman KS (2005) Versatility at the nuclear pore complex: lessons learned from the nucleoporin Nup153. Chromosoma 114:319-330

Bayliss R, Leung SW, Baker RP, Quimby BB, Corbett AH, Stewart M (2002a) Structural basis for the interaction between NTF2 and nucleoporin FxFG repeats. EMBO J 21:2843-2853 
Bayliss R, Littlewood T, Stewart M (2000) Structural basis for the interaction between FxFG nucleoporin repeats and importin-beta in nuclear trafficking. Cell 102:99-108

Bayliss R, Littlewood T, Strawn LA, Wente SR, Stewart M (2002b) GLFG and FxFG nucleoporins bind to overlapping sites on importin-beta. J Biol Chem 277:50597-50606

Beck M, Forster F, Ecke M, Plitzko JM, Melchior F, Gerisch G, Baumeister W, Medalia O (2004) Nuclear pore complex structure and dynamics revealed by cryoelectron tomography. Science 306:1387-1390

Beck M, Lucic V, Forster F, Baumeister W, Medalia O (2007) Snapshots of nuclear pore complexes in action captured by cryo-electron tomography. Nature 449:611-615

Bednenko J, Cingolani G, Gerace L (2003) Importin beta contains a $\mathrm{COOH}$-terminal nucleoporin binding region important for nuclear transport. J Cell Biol 162:391-401

Ben-Efraim I, Gerace L (2001) Gradient of increasing affinity of importin beta for nucleoporins along the pathway of nuclear import. J Cell Biol 152:411-417

Berke IC, Boehmer T, Blobel G, Schwartz TU (2004) Structural and functional analysis of Nup133 domains reveals modular building blocks of the nuclear pore complex. J Cell Biol 167:591-597

Bernad R, Engelsma D, Sanderson H, Pickersgill H, Fornerod M (2006) Nup214-Nup88 nucleoporin subcomplex is required for CRM1-mediated $60 \mathrm{~S}$ preribosomal nuclear export. J Biol Chem 281:19378-19386

Brown HG, Hoh JH (1997) Entropic exclusion by neurofilament sidearms: a mechanism for maintaining interfilament spacing. Biochemistry $36: 15035-15040$

Bustamante C, Marko JF, Siggia ED, Smith S (1994) Entropic elasticity of lambda-phage DNA. Science 265:1599-1600

Callan HG, Tomlin SG (1950) Experimental studies on amphibian oocyte nuclei. I. Investigation of the structure of the nuclear membrane by means of the electron microscope. Proc R Soc Lond B Biol Sci 137:367-378

Cronshaw JA, Krutchinsky AN, Zhang WZ, Chait BT, Matunis MJ (2002) Proteomic analysis of the mammalian nuclear pore complex. J Cell Biol 158:915-927

Danker T, Schillers H, Storck J, Shahin V, Kramer B, Wilhelmi M, Oberleithner H (1999) Nuclear hourglass technique: an approach that detects electrically open nuclear pores in Xenopus laevis oocyte. Proc Natl Acad Sci USA 96:13530-13535

Denning DP, Patel SS, Uversky V, Fink AL, Rexach M (2003) Disorder in the nuclear pore complex: the FG repeat regions of nucleoporins are natively unfolded. Proc Natl Acad Sci USA 100:2450-2455

Denning DP, Rexach MF (2007) Rapid evolution exposes the boundaries of domain structure and function in natively unfolded FG nucleoporins. Mol Cell Proteomics 6:272-282

Denning DP, Uversky V, Patel SS, Fink AL, Rexach M (2002) The Saccharomyces cerevisiae nucleoporin Nup2p is a natively unfolded protein. J Biol Chem 277:33447-33455

Devos D, Dokudovskaya S, Williams R, Alber F, Eswar N, Chait BT, Rout MP, Sali A (2006) Simple fold composition and modular architecture of the nuclear pore complex. Proc Natl Acad Sci USA 103:2172-2177

Drin G, Casella JF, Gautier R, Boehmer T, Schwartz TU, Antonny B (2007) A general amphipathic alpha-helical motif for sensing membrane curvature. Nat Struct Mol Biol 14:138-146

Dunker AK, Lawson JD, Brown CJ, Williams RM, Romero P, Oh JS, Oldfield CJ, Campen AM, Ratliff CR, Hipps KW, Ausio J, Nissen MS, Reeves R, Kang CH, Kissinger CR, Bailey RW, Griswold MD, Chiu M, Garner EC, Obradovic Z (2001) Intrinsically disordered protein. J Mol Graph Model 19:26-59

Dutta AK, Belfort G (2007) Adsorbed gels versus brushes: viscoelastic differences. Langmuir 23:3088-3094
Enss K, Danker T, Schlune A, Buchholz I, Oberleithner H (2003) Passive transport of macromolecules through Xenopus laevis nuclear envelope. J Membr Biol 196:147-155

Fahrenkrog B, Aebi U (2003) The nuclear pore complex: nucleocytoplasmic transport and beyond. Nat Rev Mol Cell Biol 4:757-766

Fahrenkrog B, Hurt EC, Aebi U, Pante N (1998) Molecular architecture of the yeast nuclear pore complex: localization of Nsp1p subcomplexes. J Cell Biol 143:577-588

Fahrenkrog B, Maco B, Fager AM, Koser J, Sauder U, Ullman KS, Aebi U (2002) Domain-specific antibodies reveal multiple-site topology of Nup153 within the nuclear pore complex. J Struct Biol 140:254-267

Feldherr CM, Akin D (1997) The location of the transport gate in the nuclear pore complex. J Cell Sci 110(Pt 24):3065-3070

Franke WW (1974) Structure, biochemistry, and functions of the nuclear envelope. Int Rev Cytol Suppl 4:71-236

Franke WW, Scheer U (1970) The ultrastructure of the nuclear envelope of amphibian oocytes: a reinvestigation. II. The immature oocyte and dynamic aspects. J Ultrastruct Res 30:317-327

Frey S, Gorlich D (2007) A saturated FG-repeat hydrogel can reproduce the permeability properties of nuclear pore complexes. Cell 130:512-523

Frey S, Richter RP, Gorlich D (2006) FG-rich repeats of nuclear pore proteins form a three-dimensional meshwork with hydrogel-like properties. Science 314:815-817

Gall JG (1967) Octagonal nuclear pores. J Cell Biol 32:391-399

Gerace L, Burke B (1988) Functional organization of the nuclear envelope. Annu Rev Cell Biol 4:335-374

Gorlich D, Kutay U (1999) Transport between the cell nucleus and the cytoplasm. Annu Rev Cell Dev Biol 15:607-660

Gorlich D, Pante N, Kutay U, Aebi U, Bischoff FR (1996) Identification of different roles for RanGDP and RanGTP in nuclear protein import. EMBO J 15:5584-5594

Gorlich D, Prehn S, Laskey RA, Hartmann E (1994) Isolation of a protein that is essential for the first step of nuclear-protein import. Cell 79:767-778

Grant RP, Hurt E, Neuhaus D, Stewart M (2002) Structure of the C-terminal FG-nucleoporin binding domain of Tap/NXF1. Nat Struct Biol 9:247-251

Grant RP, Neuhaus D, Stewart M (2003) Structural basis for the interaction between the Tap/NXF1 UBA domain and FG nucleoporins at $1 \AA$ resolution. J Mol Biol 326:849-858

Griffis ER, Altan N, Lippincott-Schwartz J, Powers MA (2002) Nup98 is a mobile nucleoporin with transcription-dependent dynamics. Mol Biol Cell 13:1282-1297

Griffis ER, Craige B, Dimaano C, Ullman KS, Powers MA (2004) Distinct functional domains within nucleoporins Nup153 and Nup98 mediate transcription-dependent mobility. Mol Biol Cell 15:1991-2002

Halperin A, Tirrell M, Lodge TP (1992) Tethered chains in polymer microstructures. Adv Polym Sci 100:31-71

Handa N, Kukimoto-Niino M, Akasaka R, Kishishita S, Murayama K, Terada $\mathrm{T}$, Inoue $\mathrm{M}$, Kigawa $\mathrm{T}$, Kose $\mathrm{S}$, Imamoto $\mathrm{N}$, Tanaka $\mathrm{A}$, Hayashizaki Y, Shirouzu M, Yokoyama S (2006) The crystal structure of mouse Nup35 reveals atypical RNP motifs and novel homodimerization of the RRM domain. J Mol Biol 363:114-124

Higa MM, Alam SL, Sundquist WI, Ullman KS (2007) Molecular characterization of the ran-binding zinc finger domain of NUP153. J Biol Chem 282:17090-17100

Hinshaw JE, Carragher BO, Milligan RA (1992) Architecture and design of the nuclear-pore complex. Cell 69:1133-1141

Hodel AE, Hodel MR, Griffis ER, Hennig KA, Ratner GA, Xu S, Powers MA (2002) The three-dimensional structure of the autoproteolytic, nuclear pore-targeting domain of the human nucleoporin Nup98. Mol Cell 10:347-358 
Hutten S, Kehlenbach RH (2006) Nup214 is required for CRM1dependent nuclear protein export in vivo. Mol Cell Biol 26:67726785

Isgro TA, Schulten K (2005) Binding dynamics of isolated nucleoporin repeat regions to importin- $\beta$. Structure 13:1869-1879

Isgro TA, Schulten K (2007a) Association of nuclear pore FG-repeat domains to NTF2 import and export complexes. J Mol Biol 366:330-345

Isgro TA, Schulten K (2007b) Cse1p-binding dynamics reveal a binding pattern for FG-repeat nucleoporins on transport receptors. Structure 15:977-991

Jaggi RD, Franco-Obregon A, Ensslin K (2003a) Quantitative topographical analysis of nuclear pore complex function using scanning force microscopy. Biophys J 85:4093-4098

Jaggi RD, Franco-Obregon A, Muhlhausser P, Thomas F, Kutay U, Ensslin K (2003b) Modulation of nuclear pore topology by transport modifiers. Biophys J 84:665-670

Kessel RG (1969) Fine structure of the pore-annulus complex in the nuclear envelope and annulate lamellae of germ cells. Z Zellforsch Mikrosk Anat 94:441-453

Kim SH, Marmo C, Somorjai GA (2001) Friction studies of hydrogel contact lenses using AFM: non-crosslinked polymers of low friction at the surface. Biomaterials 22:3285-3294

Kim SH, Opdahl A, Marmo C, Somorjai GA (2002) AFM and SFG studies of pHEMA-based hydrogel contact lens surfaces in saline solution: adhesion, friction, and the presence of non-crosslinked polymer chains at the surface. Biomaterials 23:1657-1666

Kiseleva E, Allen TD, Rutherford S, Bucci M, Wente SR, Goldberg MW (2004) Yeast nuclear pore complexes have a cytoplasmic ring and internal filaments. J Struct Biol 145:272-288

Kopito RB, Elbaum M (2007) Reversibility in nucleocytoplasmic transport. Proc Natl Acad Sci USA 104:12743-12748

Kubitscheck U, Grunwald D, Hoekstra A, Rohleder D, Kues T, Siebrasse JP, Peters R (2005) Nuclear transport of single molecules: dwell times at the nuclear pore complex. J Cell Biol 168:233-243

Lim RYH, Aebi U, Stoffler D (2006a) From the trap to the basket: getting to the bottom of the nuclear pore complex. Chromosoma 115:15-26

Lim RYH, Fahrenkrog B (2006) The nuclear pore complex up close. Curr Opin Cell Biol 18:342-347

Lim RYH, Fahrenkrog B, Koser J, Schwarz-Herion K, Deng J, Aebi U (2007a) Nanomechanical basis of selective gating by the nuclear pore complex. Science 318:640-643

Lim RYH, Huang NP, Koser J, Deng J, Lau KHA, Schwarz-Herion K, Fahrenkrog B, Aebi U (2006b) Flexible phenylalanine-glycine nucleoporins as entropic barriers to nucleocytoplasmic transport. Proc Natl Acad Sci USA 103:9512-9517

Lim RYH, Koser J, Huang NP, Schwarz-Herion K, Aebi U (2007b) Nanomechanical interactions of phenylalanine-glycine nucleoporins studied by single molecule force-volume spectroscopy. J Struct Biol 159:277-289

Liu SM, Stewart M (2005) Structural basis for the high-affinity binding of nucleoporin Nup1p to the Saccharomyces cerevisiae importinbeta homologue, Kap95p. J Mol Biol 349:515-525

Macara IG (2001) Transport into and out of the nucleus. Microbiol Mol Biol Rev 65:570-594

Maeshima K, Yahata K, Sasaki Y, Nakatomi R, Tachibana T, Hashikawa T, Imamoto F, Imamoto N (2006) Cell-cycle-dependent dynamics of nuclear pores: pore-free islands and lamins. J Cell Sci 119:4442-4451

Marko JF, Siggia ED (1995) Stretching DNA. Macromolecules 28:8759-8770

Maul GG (1971) On the octagonality of the nuclear pore complex. J Cell Biol 51:558-563

Mazzanti M, Bustamante JO, Oberleithner H (2001) Electrical dimension of the nuclear envelope. Physiol Rev 81:1-19
Melcak I, Hoelz A, Blobel G (2007) Structure of Nup58/45 suggests flexible nuclear pore diameter by intermolecular sliding. Science 315:1729-1732

Melchior F, Paschal B, Evans J, Gerace L (1993) Inhibition of nuclearprotein import by nonhydrolyzable analogs of GTP and identification of the small GTPase Ran/TC4 as an essential transport factor. J Cell Biol 123:1649-1659

Milner ST (1991) Polymer brushes. Science 251:905-914

Moore MS, Blobel G (1993) The GTP-binding protein Ran/TC4 is required for protein import into the nucleus. Nature 365:661-663

Mooren OL, Erickson ES, Moore-Nichols D, Dunn RC (2004) Nuclear side conformational changes in the nuclear pore complex following calcium release from the nuclear membrane. Phys Biol 1:125134

Mukhopadhyay R, Hoh JH (2001) AFM force measurements on microtubule-associated proteins: the projection domain exerts a longrange repulsive force. FEBS Lett 505:374-378

Nachury MV, Weis K (1999) The direction of transport through the nuclear pore can be inverted. Proc Natl Acad Sci USA 96:96229627

Naim B, Brumfeld V, Kapon R, Kiss V, Nevo R, Reich Z (2007) Passive and facilitated transport in nuclear pore complexes is largely uncoupled. J Biol Chem 282:3881-3888

Napetschnig J, Blobel G, Hoelz A (2007) Crystal structure of the N-terminal domain of the human protooncogene Nup214/CAN. Proc Natl Acad Sci USA 104:1783-1788

Paine PL, Moore LC, Horowitz SB (1975) Nuclear-envelope permeability. Nature 254:109-114

Pante N, Aebi U (1995) Toward a molecular understanding of the structure and function of the nuclear pore complex. Int Rev Cytol 162B:225-255

Pante N, Aebi U (1996) Molecular dissection of the nuclear pore complex. Crit Rev Biochem Mol Biol 31:153-199

Pante N, Kann M (2002) Nuclear pore complex is able to transport macromolecules with diameters of similar to $39 \mathrm{~nm}$. Mol Biol Cell 13:425-434

Paradise A, Levin MK, Korza G, Carson JH (2007) Significant proportions of nuclear transport proteins with reduced intracellular mobilities resolved by fluorescence correlation spectroscopy. J Mol Biol 365:50-65

Patel SS, Belmont BJ, Sante JM, Rexach MF (2007) Natively unfolded nucleoporins gate protein diffusion across the nuclear pore complex. Cell 129:83-96

Paulillo SM, Phillips EM, Koser J, Sauder U, Ullman KS, Powers MA, Fahrenkrog B (2005) Nucleoporin domain topology is linked to the transport status of the nuclear pore complex. J Mol Biol 351:784-798

Paulillo SM, Powers MA, Ullman KS, Fahrenkrog B (2006) Changes in nucleoporin domain topology in response to chemical effectors. J Mol Biol 363:39-50

Peters R (2005) Translocation through the nuclear pore complex: Selectivity and speed by reduction-of-dimensionality. Traffic 6:421-427

Radu A, Blobel G, Moore MS (1995a) Identification of a protein complex that is required for nuclear-protein import and mediates docking of import substrate to distinct nucleoporins. Proc Natl Acad Sci USA 92:1769-1773

Radu A, Moore MS, Blobel G (1995b) The peptide repeat domain of nucleoporin Nup98 functions as a docking site in transport across the nuclear-pore complex. Cell 81:215-222

Rexach M, Blobel G (1995) Protein import into nuclei-association and dissociation reactions involving transport substrate, transport factors, and nucleoporins. Cell 83:683-692

Ribbeck K, Gorlich D (2002) The permeability barrier of nuclear pore complexes appears to operate via hydrophobic exclusion. EMBO J 21:2664-2671 
Robinson MA, Park S, Sun ZY, Silver PA, Wagner G, Hogle JM (2005) Multiple conformations in the ligand-binding site of the yeast nuclear pore-targeting domain of Nup116p. J Biol Chem 280:35723-35732

Rout MP, Aitchison JD (2001) The nuclear pore complex as a transport machine. J Biol Chem 276:16593-16596

Rout MP, Aitchison JD, Magnasco MO, Chait BT (2003) Virtual gating and nuclear transport: the hole picture. Trends Cell Biol 13:622-628

Rout MP, Aitchison JD, Suprapto A, Hjertaas K, Zhao YM, Chait BT (2000) The yeast nuclear pore complex: composition, architecture, and transport mechanism. J Cell Biol 148:635-651

Sabri N, Roth P, Xylourgidis N, Sadeghifar F, Adler J, Samakovlis C (2007) Distinct functions of the Drosophila Nup153 and Nup214 FG domains in nuclear protein transport. J Cell Biol 178:557-565

Schwartz TU (2005) Modularity within the architecture of the nuclear pore complex. Curr Opin Struct Biol 15:221-226

Schwarz-Herion K, Maco B, Sauder U, Fahrenkrog B (2007) Domain topology of the p62 complex within the 3-D architecture of the nuclear pore complex. J Mol Biol 370:796-806

Shahin V, Danker T, Enss K, Ossig R, Oberleithner H (2001) Evidence for $\mathrm{Ca} 2+-$ and ATP-sensitive peripheral channels in nuclear pore complexes. FASEB J 15:1895-1901

Shulga N, Goldfarb DS (2003) Binding dynamics of structural nucleoporins govern nuclear pore complex permeability and may mediate channel gating. Mol Cell Biol 23:534-542

Soullam B, Worman HJ (1995) Signals and structural features involved in integral membrane protein targeting to the inner nuclear membrane. J Cell Biol 130:15-27

Stewart M (2007) Molecular mechanism of the nuclear protein import cycle. Nat Rev Mol Cell Biol 8:195-208

Stoffler D, Feja B, Fahrenkrog B, Walz J, Typke D, Aebi U (2003) Cryo-electron tomography provides novel insights into nuclear pore architecture: implications for nucleocytoplasmic transport. J Mol Biol 328:119-130

Stoffler D, Goldie KN, Feja B, Aebi U (1999) Calcium-mediated structural changes of native nuclear pore complexes monitored by time-lapse atomic force microscopy. J Mol Biol 287:741-752

Strawn LA, Shen TX, Shulga N, Goldfarb DS, Wente SR (2004) Minimal nuclear pore complexes define FG repeat domains essential for transport. Nat Cell Biol 6:197-206

Suntharalingam M, Wente SR (2003) Peering through the pore: nuclear pore complex structure, assembly, and function. Dev Cell 4:775-789

Terry LJ, Wente SR (2007) Nuclear mRNA export requires specific FG nucleoporins for translocation through the nuclear pore complex. J Cell Biol 178:1121-1132

Timney BL, Tetenbaum-Novatt J, Agate DS, Williams R, Zhang WZ, Chait BT, Rout MP (2006) Simple kinetic relationships and nonspecific competition govern nuclear import rates in vivo. J Cell Biol 175:579-593

Tompa P (2002) Intrinsically unstructured proteins. Trends Biochem Sci 27:527-533
Tran EJ, Wente SR (2006) Dynamic nuclear pore complexes: life on the edge. Cell 125:1041-1053

Unwin PN, Milligan RA (1982) A large particle associated with the perimeter of the nuclear pore complex. J Cell Biol 93:63-75

van Deursen J, Boer J, Kasper L, Grosveld G (1996) G2 arrest and impaired nucleocytoplasmic transport in mouse embryos lacking the proto-oncogene CAN/Nup214. EMBO J 15:5574-5583

Walther TC, Pickersgill HS, Cordes VC, Goldberg MW, Allen TD, Mattaj IW, Fornerod M (2002) The cytoplasmic filaments of the nuclear pore complex are dispensable for selective nuclear protein import. J Cell Biol 158:63-77

Wang HW, Clapham DE (1999) Conformational changes of the in situ nuclear pore complex. Biophys J 77:241-247

Wang RW, Brattain MG (2007) The maximal size of protein to diffuse through the nuclear pore is larger than $60 \mathrm{kDa}$. FEBS Lett 581:3164-3170

Weirich CS, Erzberger JP, Berger JM, Weis K (2004) The N-terminal domain of Nup159 forms a beta-propeller that functions in mRNA export by tethering the helicase Dbp5 to the nuclear pore. Mol Cell 16:749-760

Weis K (2003) Regulating access to the genome: nucleocytoplasmic transport throughout the cell cycle. Cell 112:441-451

Weis K (2007) The nuclear pore complex: oily spaghetti or gummy bear? Cell 130:405-407

Winey M, Yarar D, Giddings TH Jr, Mastronarde DN (1997) Nuclear pore complex number and distribution throughout the Saccharomyces cerevisiae cell cycle by three-dimensional reconstruction from electron micrographs of nuclear envelopes. Mol Biol Cell 8:2119-2132

Wozniak RW, Rout MP, Aitchison JD (1998) Karyopherins and kissing cousins. Trends Cell Biol 8:184-188

Yang Q, Rout MP, Akey CW (1998) Three-dimensional architecture of the isolated yeast nuclear pore complex: functional and evolutionary implications. Mol Cell 1:223-234

Yang WD, Gelles J, Musser SM (2004) Imaging of single-molecule translocation through nuclear pore complexes. Proc Natl Acad Sci USA 101:12887-12892

Yang WD, Musser SM (2006) Nuclear import time and transport efficiency depend on importin beta concentration. J Cell Biol 174:951-961

Zeitler B, Weis K (2004) The FG-repeat asymmetry of the nuclear pore complex is dispensable for bulk nucleocytoplasmic transport in vivo. J Cell Biol 167:583-590

Zhao B, Brittain WJ (2000) Polymer brushes: surface-immobilized macromolecules. Prog Polym Sci 25:677-710

Zilman A, Di Talia S, Chait BT, Rout MP, Magnasco MO (2007) Efficiency, selectivity, and robustness of nucleocytoplasmic transport. PLoS Comput Biol 3:1281-1290

Zimmerman SB, Minton AP (1993) Macromolecular crowding-biochemical, biophysical, and physiological consequences. Annu Rev Biophys Biomol Struct 22:27-65 